\title{
Rapid Allergen Inactivation Using Atmospheric Pressure Cold
}

\section{Plasma}

Yan $\mathrm{Wu}^{1, \#, \&}$, Yongdong Liang ${ }^{2,3, \#}$, Kai Wei ${ }^{1}, \mathrm{Wei}^{2} \mathrm{i}^{2,3}$, Maosheng $\mathrm{Yao}^{1, *}$, and Jue Zhang $2,3, *$

\footnotetext{
${ }^{1}$ State Key Joint Laboratory of Environmental Simulation and Pollution Control, College of Environmental Sciences and Engineering, Peking University, Beijing 100871, China

${ }^{2}$ Department of Biomedical Engineering, College of Engineering, Peking University, Beijing 100871, China

${ }^{3}$ Academy for Advanced Interdisciplinary Studies, Peking University, Beijing 100871, China
}

Environmental Science and Technology

*Corresponding authors:

Maosheng Yao, Email: yao@pku.edu.cn, Ph:+86 0106276 7282; Fax: +860106276 7282

State Key Joint Laboratory of Environmental Simulation and Pollution Control, College of Environmental Sciences and Engineering, Peking University, Beijing 100871, China Jue Zhang, Email: zhangjue@pku.edu.cn, Ph: +86 010 62755036; Fax: +86 010 62755036

Department of Biomedical Engineering, College of Engineering, Peking University, Beijing 100871, China

\#: Y. Wu and Y. Liang contributed equally to the work; \&: Y. Wu is presently affiliated with SINBERBEST Program, Berkeley Education Alliance for Research in Singapore (BEARS) and School of Civil and Environmental Engineering, Nanyang Technological University, 50 Nanyang Avenue 639798, Singapore

\section{Beijing}

Feb 3, 2014 


\section{Supporting Information}

\section{File S1: Descriptions of the other plasma device used for liquid samples in this work}

Fig S1 (A) shows the picture of micro-jet device, Fig S1 (B) shows the generated plasma torch which was round $30{ }^{\circ} \mathrm{C}$ with a length of $1.5 \mathrm{~cm}$, and Fig S1 (C) shows the dimensions of this system. For the micro-jet plasma device, two metal electrodes were separated from each other by a dielectric layer of $0.5 \mathrm{~mm}$ thickness. The openings in the two electrodes are $0.8 \mathrm{~mm}$ in diameter. One electrode was completely embedded in the device and powered by a direct current (DC) power supply (Matsusada AU5R120, Japan) and the other electrode was grounded for safety. Although both positive and negative high voltages were able to generate and maintain the plasma flam/torch, a negative high voltage was chosen in this work. A ballast resistor at a voltage of $5 \mathrm{kV}$ followed by a current monitoring resistor of 100 $\mathrm{kV}$ was inserted between the embedded electrode and DC power supply. The discharge sustaining voltage was around 400-800 V with an operating current in the range of 20-35 mA. The flow rate of working gas carrier used for the liquid samples was $5 \mathrm{~s} / \mathrm{m}$ (Standard Liter per Minute). Under these operating conditions, a plasma jet of $15 \mathrm{~mm}$ can be totally generated in the liquid as shown in Fig S1 (B). To our best knowledge, the micro-jet system in which plasma was generated entirely in the liquid was the first successful attempt to be achieved in the literature and could be employed to decontaminate liquid samples of different depth. 


\section{File S2 Allergens inactivation by UV exposure and water-bath heating}

In this study, the efficiencies of two common physical methods such as ultraviolet (UV) irradiation and water-bath heating which are often practiced as germicide and virucide were also investigated and compared with the APCP for inactivating the waterborne allergens. Here, the dog allergen Can $f 1$ was used as an example. When performing the allergen inactivation experiments using ultraviolet irradiation, $300 \mu \mathrm{L}$ of diluted allergen standard was pipetted into the wells of a 96-well plate with three replicates. Then the allergen suspensions were exposed to ultraviolet irradiation provided by the UV lamp equipped with a BioSafety cabinet (NuAire, Inc.) for varying exposure times ( $15 \mathrm{~min}, 30 \mathrm{~min}$ and $60 \mathrm{~min}$ ). According to a study, it takes about 12.5 minutes to allow a UV dose of $40 \mu \mathrm{W} / \mathrm{cm}^{2}$, which is a minimum requirement for a BioSafety cabinet, to achieve the killing of spores (48). In this study, wet heating supplied by a digital water bath (LWB-22T, Labnics Equipment Inc., Fremont, California, USA) was employed. $500 \mu \mathrm{L}$ of diluted allergen standard was added into a $1.5 \mathrm{~mL}$ tube (Corning ${ }^{\circledR}$ Premium Quality, Acton, MA) with three replicates and then exposed to water bath heating at different temperature $\left(60^{\circ} \mathrm{C}\right.$ and $\left.100^{\circ} \mathrm{C}\right)$ for $30 \mathrm{~min}$. The allergenicities of these allergen samples were further analyzed using double antibody sandwich ELISA method. In our study, the experiments for both control and treated were independently conducted three times and were carried out in a BioSafety Level II cabinet (NuAire, Inc.). 


\section{Supporting Figures}

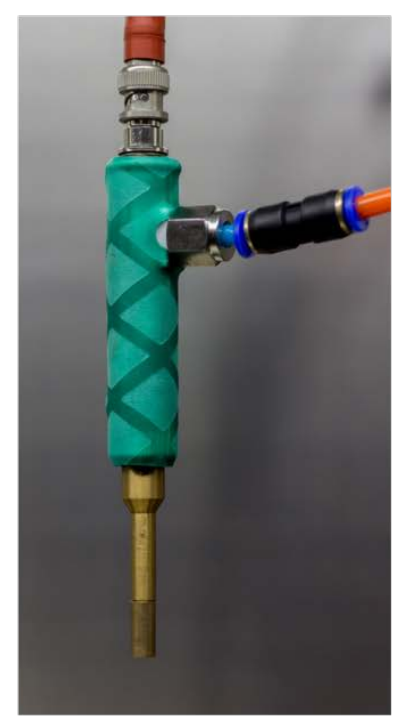

A. Micro-jet system

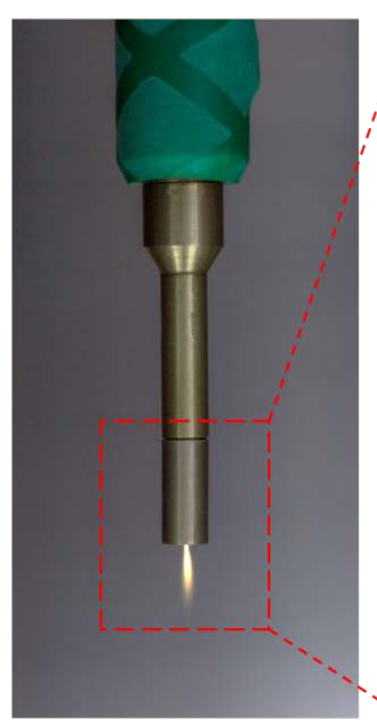

B. Excited Micro-jet system

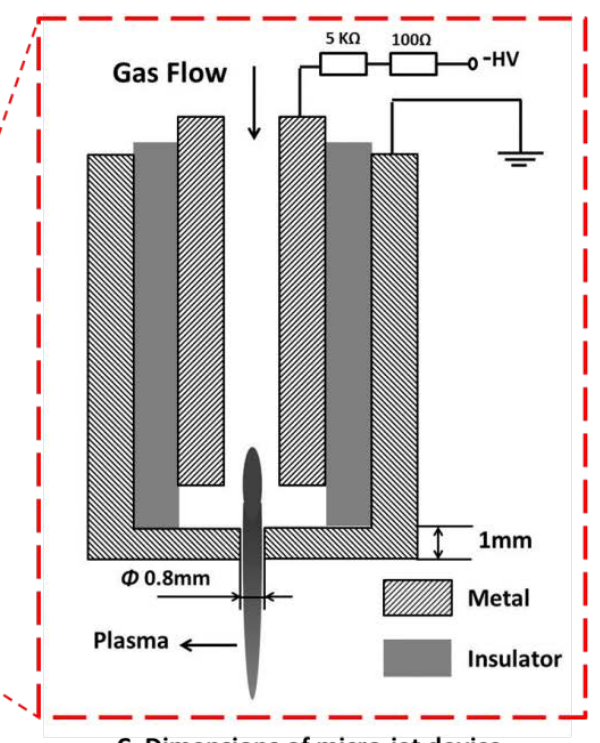

C. Dimensions of micro-jet device

Fig S1 A) The DC atmospheric-pressure cold plasma micro-jet system for treating liquid samples; B) Excited micro-jet system with $10-\mathrm{mm}$ plasma torch produced at a voltage of between $400-800 \mathrm{~V} ; \mathrm{C}$ ) the high resolution schematic details of the micro-jet as depicted in B). 


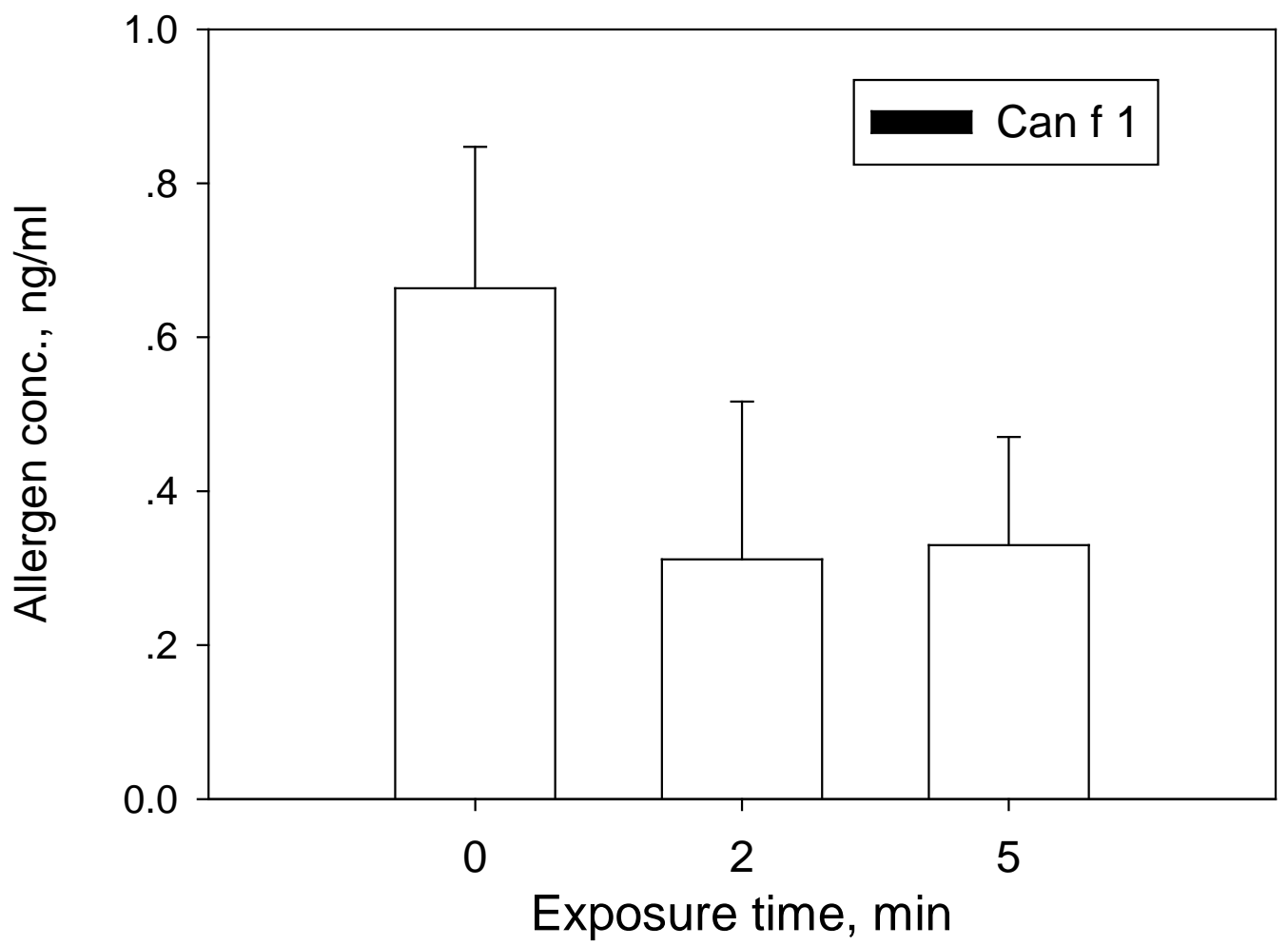

Fig S2 Inactivation of water-borne dog allergen Can $f 1$ using atmospheric cold plasma produced by the device shown in Fig S1 using indoor air at the energy level of $28 \mathrm{~W}$ for different time periods; data points and error bars represent averages and standard deviations from three independent repeats. 


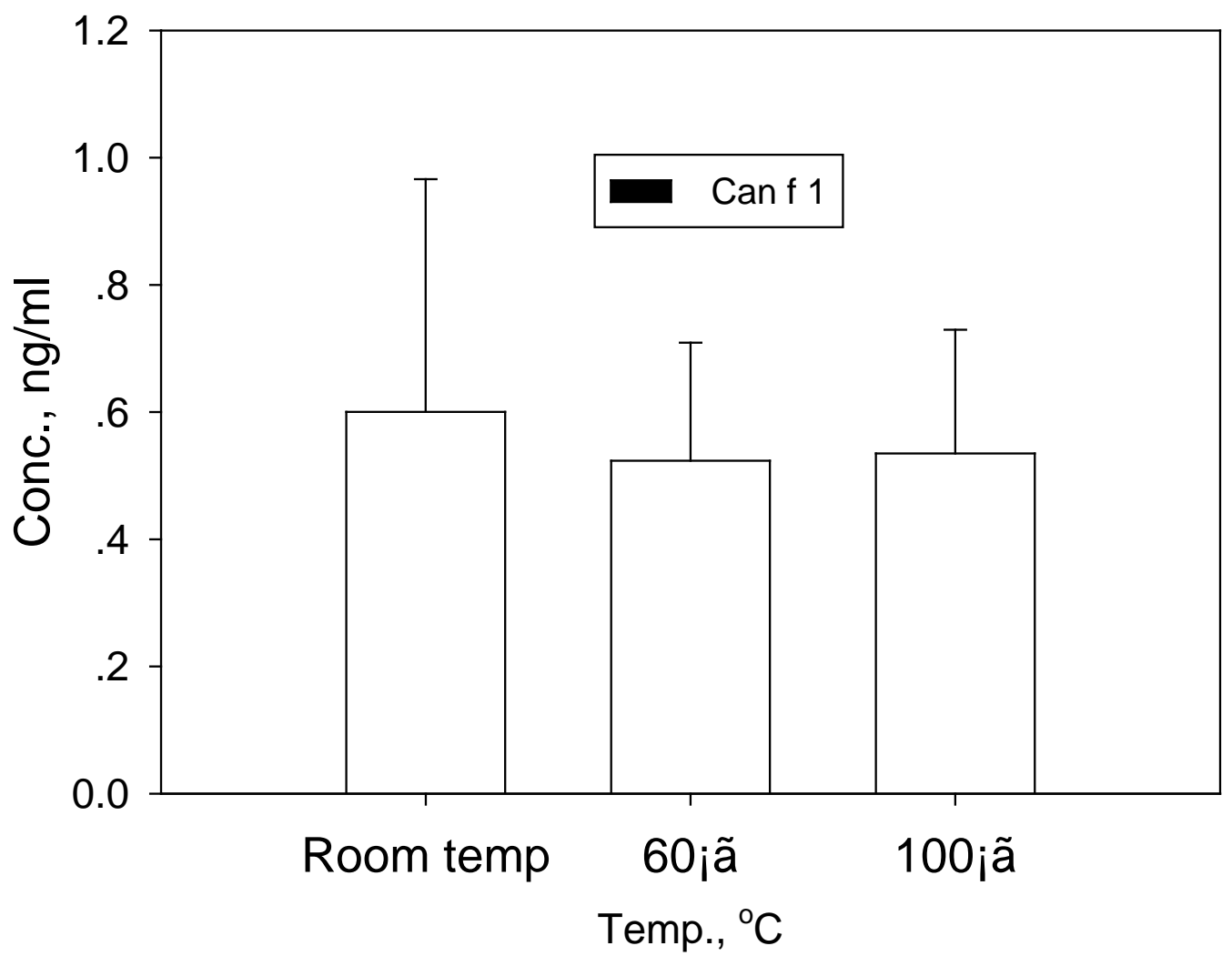

Fig S3 Effects of different temperatures on the allergenicity of water-borne dog allergen Can $\mathrm{f} 1$ (30 min exposure); data points and error bars refer to averages and standard deviations of three independent experiments (not statistically significant; p-value $=0.33)$. 


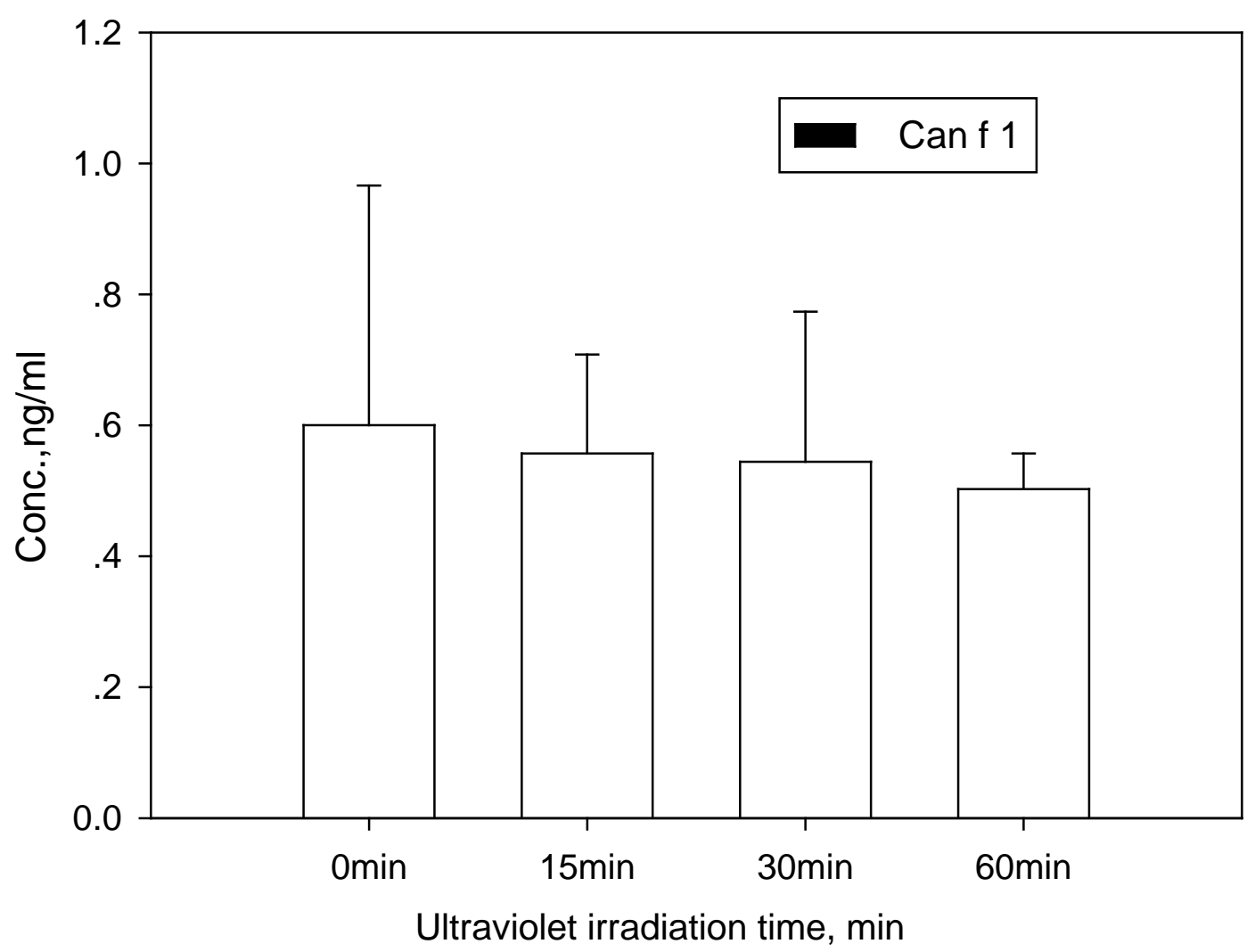

Fig S4 Effects of ultraviolet irradiation on water-borne dog allergen Can $f 1$ for different time periods (not statistically significant; $p$-value=0.38); UV was provided by the equipped lamp with BioSafety cabinet; data points and error bars represent averages and standard deviations of three independent repeats. 
Liquid-borne allergens: ELISA Assay

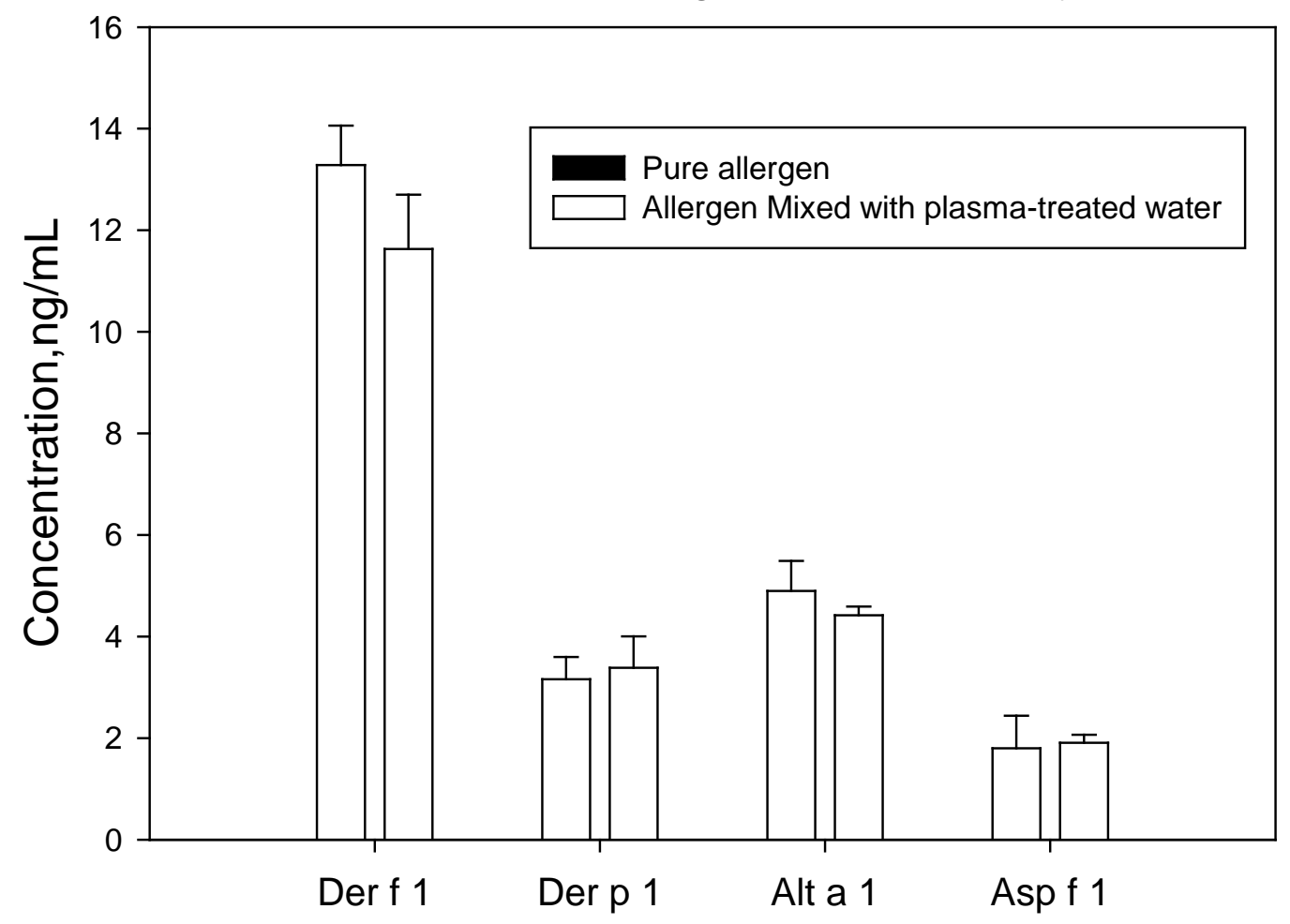

Fig S5 Effects of plasma-treated DI (deionized) water on the ELISA assay for detecting allergens (Der $f$ 1, Der $p$ 1, Alt a 1 and Asp $f$ 1); plasma was produced at the energy level of $28 \mathrm{~W}$ using the device shown in Fig S1; data points and error bars represent averages and standard deviations of three independent repeats. 